(107/702) of men were infected with CT and/or NG, $8.4 \%$ $(33 / 392)$ at 6 months, and $15.6 \%(10 / 64)$ at 12 months. The incidence of anorectal and/or urogenital infection was 6.0 per 100 person-years (P-Y), and 4.6 per 100 P-Ys for urogenital infection alone. Increased risk of urogenital infection was associated drug use in the past year $(\mathrm{aHR}=2.44 ; 95 \% \mathrm{CI}: 1.17-$ 5.08), versatile (compared to insertive) usual sexual positioning $(\mathrm{aHR}=2.40 ; 95 \% \mathrm{CI}: 1.01-5.71)$ or water-based lubricant use compared to no lubricant use $(\mathrm{aHR}=5.72$; 95\% CI: $1.28-$ 25.5). Protective factors $(\mathrm{p}<0.10$ each) included increasing age $(\mathrm{aHR}=0.94)$, condom use at last sex $(\mathrm{aHR}=0.53)$, and increasing social support $(\mathrm{aHR}=0.73$ per quartile increase). Child abuse scores, depressive symptom measures, HIV status, and alcohol use were not associated with incidence.

Conclusions NG and/or CT and incidence was high despite baseline testing and treatment, quarterly visits, and peer counselling and support for reducing HIV risk. Partner treatment and program exposure measures will be analysed as data accrual completes with follow-up continuing to September 2017.

\subsection{FEASIBILITY, ACCEPTABILITY AND POTENTIAL ROLE OF PREP IN COMBINATION HIV PREVENTION FOR MSM AND TRANSWOMEN IN PERU: RESULTS OF A MIXED- METHODS STUDY}

${ }^{1}$ Carlos F Cáceres, ${ }^{2}$ Annick Borquez, ${ }^{1}$ Aron Nunez-Curto, ${ }^{1}$ Ximena Salazar, ${ }^{1}$ Juan V Guanira, ${ }^{1} A$ Alfonso Silva-Santisteban, ${ }^{1}$ Angelica Motta, ${ }^{3}$ Patricia Bracamonte, ${ }^{4}$ Byelca Huamán, ${ }^{5}$ Patricia Caballero. ${ }^{1}$ Universidad Peruana Cayetano Heredia, Peru; ${ }^{2}$ University of California, San Diego, USA; ${ }^{3}$ UNAIDS; ${ }^{4}$ Ministry of Health of Peru; ${ }^{5}$ National Institute of Health of Peru

\subsection{6/sextrans-2017-053264.49}

Introduction Despite progress in treatment, HIV incidence among men who have sex with men and transwomen (MSM/ TW) remains high in Peru due to low coverage and insufficiency of HIV prevention services. In 2014-2015 a study gathered evidence for implementing combination HIV prevention for MSM/TW in collaboration with the health sector and civil society.

Methods In 6 cities, a mixed-methods study: (1) identified stakeholders' (users, providers) perspectives on existing and novel (pre-exposure prophylaxis [PrEP], Treatment as Prevention [TasP]) HIV prevention methods; (2) assessed health systems ' needs and conditions, and (3) used a previously developed mathematical model to estimate impact and costeffectiveness of combinations of 5 interventions ( 2 behavioural, 2 treatment-focused, PrEP) to reduce HIV incidence among MSM/TW in general and TW sex workers in particular. A National Consultation on Combination Prevention allowed for discussion of preliminary findings.

Results According to the stakeholders analysis, information on new combination prevention tools was limited among communities and providers alike, particularly for TasP (as PrEP trials had taken place here); misconceptions led to fear/resistance to change. Health facilities required improvements (lab access, training) to respond to new needs. The specific TW sex worker model predicted higher effectiveness for various combinations of prevention strategies. In PrEP-contaning scenarios, PrEP made a distinct contribution, yet cost-effectiveness was largely determined by drug cost. It seemed higher if PrEP was used on a smaller group at higher risk. (MSM/TW analysis is ongoing). The National Consultation showed increasing interest in PrEP/TasP among potential users and providers.
Conclusion Focused PrEP use may play a significant role in combined HIV prevention in Peru if TDF-FTC is obtained at reasonable cost.

\subsection{CHANGES IN SEXUAL RISK BEHAVIOUR AMONG DAILY PREP USERS AFTER 6 MONTHS OF USE IN THE AMSTERDAM PREP PROJECT}

${ }^{1}$ Anna Van Laarhoven, ${ }^{1}$ Elske Hoornenborg, ${ }^{1}$ Roel Achterbergh, ${ }^{2}$ Henry De Vries, ${ }^{1}$ Maria Prins, ${ }^{1}$ Maarten Schim Van Der Loeff. 'Department of Infectious Diseases, Public Health Service of Amsterdam, The Netherlands; ${ }^{2}$ Department of Dermatology, Academic Medical Centre, University of Amsterdam, The Netherlands

\subsection{6/sextrans-2017-053264.50}

Introduction Pre-exposure prophylaxis (PrEP) is an effective prevention measure against HIV. Risk compensation may partially counteract the public health effect of PrEP if this might increase STI incidence. We studied changes in sexual behaviour among men who have sex with men (MSM) and transgender women (TGW) who started daily PrEP In the Amsterdam PrEP (AMPrEP) demonstration project over the first 6 months.

Methods Participants completed a questionnaire at baseline and at the 6 mo visit, about sexual behaviour in the preceding 3 mo. At baseline information about demographics and drug use was collected. Sexual behaviour questions concerned frequency of sex, condom use and sexual position (insertive/ receptive) by partner type (casual/steady). Reported sexual behaviour at 6 mo was compared to behaviour at baseline using signed rank tests. Logistic regression was used to identify predictors for an increase in receptive condomless anal sex acts (rCAS) with casual partners.

Results In 2015-2016, 273 participants started daily PrEP. From this analysis 49 participants were excluded because they switched to intermittent PrEP $(n=23)$, were not due for their 6 months visit yet $(n=17)$, or because of missing data $(n=9)$. The total no. of sex partners (median=15) and no. of anal sex acts (median $=24)$ remained unchanged $(p=0.2, p=0.4$, respectively). The no. of casual partners increased (median from 14 to $15, p=0.03)$. The total no. of rCAS increased from a median of 3 to a median of $8(\mathrm{p}<0.001)$. The same trend was seen for rCAS with casual partners (median from 2 to $5, \mathrm{p}<0.001)$. In multivariable analysis, age $\geq 35 \mathrm{y}(\mathrm{p}=0.058)$ and chemsex $(\mathrm{GHB} / \mathrm{GBL}$, mephedrone, crystallised methamphetamine $)(p=0.003)$ were associated with an increase in rCAS with casual partners.

Conclusion During the first 6 mo of daily PrEP use, we observed an increase in sexual risk behaviour among MSM and TGW. PrEP users aged $\geq 35 \mathrm{y}$ and those engaging in chemsex were more likely to report an increase in high risk sexual behaviour. Whether risk compensation leads to an increase in STI incidence needs to be closely monitored.

\section{O09.4 ASSESSMENT OF CLINIC AND COMMUNITY RECRUITED YOUNG AFRICAN AMERICAN WOMEN FOR PREP ELIGIBILITY IN ATLANTA, GEORGIA}

${ }^{1}$ Jessica Sales, ${ }^{2}$ Anandi Sheth, ${ }^{1}$ Riley Steiner, ${ }^{3}$ Jennifer Brown, ${ }^{4}$ Andrea Swarzendruber, ${ }^{2}$ Carrie Cwiak, ${ }^{2}$ Lisa Haddad, ${ }^{2}$ Anar Patel. ${ }^{1}$ Emory University, Rollins School of Public Health, USA; ${ }^{2}$ Emory University, School of Medicine, USA; ${ }^{3}$ University of Cincinnati, College of Medicine, USA; ${ }^{4}$ University of Georgia, College of Public Health, USA

10.1136/sextrans-2017-053264.51 
Introduction HIV disproportionately impacts African Americans (AAs) in the US. Atlanta has been identified as an HIV 'hot spot' for AA women and ranks 8th in the US with new infections. Yet little is known about PrEP eligibility or interest among young AA women in Atlanta.

Methods 1261 sexually active young AA women (14-24 years) provided baseline data on self-reported sexual behaviour and laboratory-confirmed STI testing (Chlamydia (CT) and gonorrhoea (GC)) prior to participating in an HIV prevention trial in Atlanta. A convenience sample of women were recruited from 2 settings: community venues $(n=560$, ages $18-24$, 2012-2014) and sexual health clinics ( $n=701$, ages 14-20, 2005-2008) from sexual health clinics. An HIV risk index capturing key HIV risk factors for women was calculated from the self-report data. For the clinic sample the index included recent (past 90 days) condomless vaginal sex, condomless anal sex, sex with partner who has had male partners, sex while high (self), sex while high (partner), and intimate partner violence (IPV) (range: 0-6). For the community sample, the index included condomless sex at last sex, exchanged sex for goods and experienced IPV in past 90 days (range: 0-3). A single item assessed PrEP interest in the community sample only.

Results Bacterial STI positivity, a primary indicator for PrEP eligibility, was $20.5 \%(17.1 \%$ CT, 6.3\% GC) and $20.9 \%$ (18.8\% CT, 5.2\% GC) for the clinic and community samples, respectively. Of the 144 STI positive women from the clinic sample, HIV risk index scores ranged from 0-4, with $21.5 \%$ reporting no other HIV risk indicators, 31\% had one, $27.8 \%$ two, $16.7 \%$ three and $2.8 \%$ had 4 additional indicators. Of the 117 STI positive women from the community sample, HIV risk index scores ranged from $0-3$, with $51.3 \%$ reporting no other indicators, $36.8 \%$ one, $10.3 \%$ two and $1.7 \%$ had all 3 additional indicators. Among STI positive women, the most common HIV risk indicator was condomless vaginal sex, but $23.8 \%$ and $13.2 \%$ (only reported by those $>18$ years) of the community and clinic samples, respectively, reported recent IPV. $57.8 \%$ of the community sample reported they would be likely or very likely to use PrEP if available.

Conclusion Our findings indicate that young AA women in Atlanta, whether sampled from community venues or sexual health clinics, are at substantial risk for HIV and meet several PrEP eligibility criteria. Scaling up PrEP among women in Atlanta could have significant implications for HIV in this high burden region.

\subsection{PREP AWARENESS, ELIGIBILITY, AND ACCEPTABILITY AMONG HETEROSEXUALS RECRUITED FROM COMMUNITY-BASED HIV TESTING SITES}

${ }^{1}$ Alexis M Roth, ${ }^{1}$ Marisa A Felsher, ${ }^{1}$ Ana Martinez-Donate, ${ }^{2}$ Douglas Krakower, ${ }^{3}$ Zsofia Szep. ${ }^{1}$ Dornsife School of Public Health Department of Community Health and Prevention, Drexel University, USA; ${ }^{2}$ Division of Infectious Diseases, Beth Israel Deaconess Medical Centre, USA; Division of Infectious Diseases and HIV, Drexel University College of Medicine, USA

\subsection{6/sextrans-2017-053264.52}

Introduction Studies demonstrate pre-exposure prophylaxis (PrEP) can decrease HIV transmission among heterosexual men and women. To inform targeted promotional campaigns, it is necessary to identify those most likely to benefit from this intervention and assess their knowledge and attitudes towards PrEP.
Methods We recruited individuals $>18$ years old from 4 community-based rapid HIV counselling and testing sites in Philadelphia. They completed a brief survey and screener for PrEP eligibility. Eligibility was based on reporting "yes" to: sex with a partner of unknown or HIV positive status, sex in exchange for drugs/money, recent sexually transmitted infection, or syringe sharing. Chi-squared tests were used to examine factors associated with eligibility, knowledge, and attitudes among 111 self-identified heterosexuals.

Results The sample is $61 \%$ male, with race/ethnicity of Black (48\%), Latino (40\%), White (9\%) and other (3\%), and median age 44. 31\% screened PrEP eligible. Among males and females, eligibility was associated with reporting sex exchange, sex with persons of unknown HIV status, and recent STI (all $\mathrm{p}<0.05)$. By race/ethnicity, only syringe sharing differs significantly: $41.7 \%$ of Latinos, $42.9 \%$ of White participants and no Black or Other participants $(\mathrm{p}<0.04)$ reported sharing. Only $24 \%$ of participants had heard of PrEP; knowledge did not vary by group. A sizable minority reported concerns about side effects (44\%) and talking to a doctor about PrEP (30\%). However, 23\% were extremely likely or somewhat likely (30\%) to use PrEP to prevent HIV in the future.

Conclusion We identified a diverse and at-risk heterosexual population within community-based HIV counselling and testing. Most participants were unaware of PrEP, signifying promotional information is not reaching this population. Having rapid HIV testers screen for PrEP eligibility and educate clients about PrEP may be an effective way to increase PrEP awareness and acceptability among heterosexuals.

\subsection{INTEGRATION OF PREP IN AN ACADEMIC ADOLESCENT CLINIC \& IMPACT OF PREP USE ON SEXUALLY TRANSMITTED INFECTION (STI) RATES}

Renata Sanders, Miles K Oliva, Anthony Morgan, Heather Douglas, Christopher Reed, James Conley, Kathy Tomaszewski, Errol Fields, Noah Wheeler, Arik K Marcell. Johns Hopkins University, Baltimore, USA

\subsection{6/sextrans-2017-053264.53}

Introduction US youth are disproportionately affected by HIV. Pre-exposure prophylaxis (PrEP) significantly reduces the risk of HIV infection and may impact sexual risk. Clinics that serve adolescents are one site proposed in reaching sexually active youth. We sought to: 1) describe the integration of PrEP into an adolescent clinic; and 2) examine impact of PrEP use on sexually transmitted infection (STI) rates.

Methods Over 6 months, we integrated PrEP program into current family planning (FP) and HIV testing programs in an urban adolescent clinic. Patient lists were reviewed daily to identify those eligible for HIV testing/FP. Each eligible youth was asked about awareness of and interest in PrEP. We reviewed each chart to examine the PrEP cascade including: awareness, receipt of information, referral and referral acceptance, and PrEP use. We then examined whether PrEP use impacted rates of STI, comparing proportion of youth on PrEP with an STI in the 6 months before and after PrEP.

Results 234 youth were approached as part of the the HIV/FP program. The mean age was 17.7 (S.D. 3.0), 232 were black (99\%), 101 were males (43\%), 133 were females (57\%), 2 transgender (1\%), and 24 self-identified as lesbian, gay, or bisexual (LGB) (10\%). Among the 234 youth seen, $17(7.2 \%)$ were aware of PrEP, 49 (21\%) received information, and 33 (14\%) were referred for PrEP. Among those referred, 24 